\title{
Juegos y gamificación en las aulas de música de educación primaria
}

\author{
Games and gamification in the primary school music classrooms
}

\author{
iD Ana María Botella Nicolás; ana.maria.botella@uv.es \\ Esperanza Cabañero Castillo; esperanza.5493@gmail.com \\ Universitat de València (España)
}

\section{Resumen}

En la última década se refuerza la tendencia de buscar herramientas innovadoras y motivadoras que ayuden a optimizar el proceso de adquisición de aprendizajes. En esta línea, tras analizar recientes publicaciones y búsquedas en bases de datos, se señalan los juegos y la gamificación como herramientas educativas de apogeo en la actualidad. Desde un paradigma positivista, la pretensión del estudio es contrastar estos resultados con la realidad educativa en la que viven dichas estrategias dentro del aula de música de educación primaria, tomando como fuente de análisis las percepciones y experiencias de los docentes que están en activo en l'Horta Oest de Valencia. Para ello, se valora cuantitativamente la repercusión que tienen los juegos y la gamificación en la praxis docente actual, según los resultados obtenidos mediante cuestionarios. Tras el análisis se observa una destacable presencia de los juegos entre las técnicas educativas más utilizadas para trabajar y evaluar contenidos, sobre todo frente a otras técnicas consideradas tradicionales. No obstante, respecto a la gamificación, su uso e interés por parte de los maestros desciende, además de hallarse falsos conocimientos generalizados que desembocan en una insuficiente formación para poder llevarla a las aulas de manera exitosa.

Palabras clave: Juegos, gamificación, educación primaria, educación musical, estrategias educativas

\begin{abstract}
Over the past decade, the trend of discovering innovative and motivating tools to help optimize the learning process has been reinforced. After analyzing recent publications and searches in databases along these lines, games and gamification are pointed out as the educational tools of apogee today. From a positive paradigm, the aim of the study is to contrast these results with the educational reality in which these strategies exist within the Music Classroom in Primary Education, taking as a source of analysis the perceptions and experiences of teachers who are currently teaching in I'Horta Oest in Valencia. To this end, the impact of games and gamification on the teaching practice is assessed quantitatively, in accordance with the results obtained through questionnaires. After the analysis, a remarkable presence of the games is observed among the most used educational techniques to work and evaluate contents, especially compared to other techniques considered traditional. However, with regard to gamification, its use and interest on the part of teachers is decreasing, in addition the finding of widespread false knowledge results in inadequate teacher training which makes it difficult to function successfully in the classroom.
\end{abstract}

Keywords: Games, Gamification, Primary Education, Musical Education, Educational Strategies. 


\section{INTRODUCCIÓN}

Con el paso de los años, los gustos y las rutinas de las personas se van modificando, por eso la necesidad de que los docentes se mantengan formados y renueven las metodologías empleadas en el aula, de forma que se puedan adaptar a las exigencias del momento. Como recalca Montero "la educación también debe hacerse anuente a los cambios que vive la sociedad" (2017, p.76). Y, ¿̇por qué apostar por el juego?

Existen diversas teorías sobre el juego que se llevan desarrollando desde la segunda mitad del siglo XIX que ayudan a comprender el sentido del juego en la rutina de las personas. Un ejemplo sería la teoría de la dinámica infantil en el cual justifica que los niños juegan debido a su esencia infantil, o la teoría de la relajación en el que el juego aparece como actividad para recuperar la energía después de un esfuerzo. Gallardo-López y Gallardo-Vázquez (2018) en sus estudios recogen los aspectos más relevantes de estas teorías. Del mismo modo, entre las funciones esenciales del juego y, que acompañan a estas teorías, se destaca la de desarrollar de manera natural la parte creativa del cerebro, sin ser necesario un aprendizaje previo y que conjuntamente es suscitada por los intereses personales (Meneses y Monge, 2001), y la de desarrollar capacidades físicas, la comprensión, la posibilidad de adquirir conocimientos y ser consciente del mundo que nos rodea (Gallardo y Fernández, 2010; Gómez-Martín, GómezMartín, y González-Calero, 2004; Montero, 2017). Se puede confirmar, entonces, que la acción de jugar tiene innumerables beneficios psicomotores y psicológicos que, además de ser un entretenimiento, trasladados al ámbito educativo podrían contribuir en el proceso de enseñanzaaprendizaje (Morillas, 2016) y potenciar que este sea más significativo (de Soto, 2018).

En base a esta afirmación se llega al fenómeno de la gamificación, una de las herramientas motivadoras que surgen del juego y que asciende paulatinamente en las búsquedas que se realizan en las bases de datos de Scopus y Google Scholar, entre otras (Hamari, Koivisto y Sarsa, 2014). I-Help, Re-Mision y System Greenifi son algunos ejemplos de las numerosas aplicaciones de gamificación que se pueden encontrar dentro del ámbito educativo (Díaz y Troyano, 2013). Profundizando en el concepto, Zichermann y Cunninhgam definen la gamificación como "un proceso relacionado con el pensamiento del jugador y las técnicas de juego para atraer a los usuarios" (2011, p.11). Y, en esta línea, Kapp la especifica como "la utilización de mecanismos, la estética y el uso del pensamiento de los juegos, para atraer a las personas, incitar a la acción, promover el aprendizaje y resolver problemas" (2012, p.9). Dicho de otra manera, se trata de transportar las mecánicas y ambientaciones de los juegos que hacen que el individuo se interese por el mismo, a otro ámbito no lúdico para generar la misma respuesta que con los juegos. 
Sin embargo, cabe enfatizar que no todos los juegos sirven en la didáctica, tal y como expresa $G^{1} e^{1}$ en una entrevista realizada por Jenkins, "los juegos (en el aula) pueden ser buenos, malos o indiferentes en función de cómo se utilizan (...) Los niños aprenden con juegos cuando participan en interacciones bien diseñadas y atractivas" (2011, párr. 4).

No obstante, y a pesar de tener una presencia notoria y encontrar estudios que nos muestran experiencias y ejemplos de aplicaciones de juegos y gamificación en el ámbito educativo (TorresToukoumidis y Romero-Rodríguez, 2018; Manzano y Domínguez, 2018), es escasa la documentación que se encuentra sobre las percepciones y aplicaciones que hacen los maestros y maestras en la etapa de educación primaria y, más en concreto, en las aulas de música. Así pues, surge la siguiente cuestión: ¿Cuál es la realidad educativa de los juegos y la gamificación dentro de las aulas de música según la praxis docente?

Para dar respuesta a esta pregunta se marcan tres objetivos principales:

1. Comparar el uso de los juegos, como herramienta de desarrollo y evaluación de los contenidos curriculares de música, frente a la aplicación de otras herramientas.

2. Manifestar la situación actual en la que se encuentran los docentes especialistas de música con referencia a la gamificación. Formación, conocimientos y aplicaciones.

3. Analizar los intereses y limitaciones que encuentran los docentes a la hora de emplear nuevas metodologías.

Las hipótesis que se plantean son las siguientes:

- Los juegos como técnica para trabajar contenidos tienen una presencia relevante dentro de las aulas de música, sin embargo, su uso desciende cuando el objetivo es evaluar.

- Existen lagunas y desconocimiento al respecto del concepto y de las posibilidades que ofrece la gamificación dentro del aula.

- Los docentes tienen interés por conocer y aplicar la gamificación en el aula de música, no obstante, la falta de formación es el gran hándicap para plantearse nuevas metodologías.

\section{MÉTODO}

El desarrollo del estudio se ha llevado a cabo desde un paradigma positivista, ya que pretende explicar la situación en la que se encuentran, actualmente, los juegos y la gamificación en un territorio específico; I’Horta Oest de Valencia. Al mismo tiempo, predecir cuál es el interés y el uso de estas metodologías dentro de las aulas de música de primaria. Por otra parte, debido a

\footnotetext{
1 James Paul Gee (1948) pedagogo relevante entre los nuevos modelos pedagógicos, quien sostiene la idea de que los juegos bien diseñados deben ser la alternativa a las prácticas escolares actuales.
} 
que el instrumento principal que se ha utilizado para llevar a cabo el estudio ha sido el cuestionario, se puede confirmar que el tipo de análisis de resultados es cuantitativo.

El cuestionario fue diseñado en las dos lenguas vehiculares del territorio (castellano y valenciano) y en los dos formatos que resultan más prácticos para el estudio: formato online y formato tangible. Además, en la portada se incluía una breve introducción con la explicación del estudio y un código QR (con instrucciones de uso) para facilitar la participación de los docentes en cualquiera de los dos formatos. En el cuerpo del cuestionario se formulan 18 preguntas, 17 con carácter cerrado (con posibilidad de añadir otra opción a las presentadas) y una pregunta final de carácter abierto.

Las variables de clasificación que se proponen son: la localidad, el tipo de centro en el que trabajan (público, privado o concertado), la categoría profesional (funcionario de carrera, interino, maestra/o titular o sustituto), la edad, el género y los años de docencia como maestros especialistas. De esta manera, comprobar si existen diferencias significativas entre los resultados de dichas variables.

En cuanto a las variables independientes que se plantean son dos, la primera es la praxis docente a nivel general, es decir, las metodologías que se utilizan dentro del aula para trabajar contenidos y evaluar. En el segundo bloque de preguntas se diseñaron cuestiones sobre gamificación; el conocimiento (o desconocimiento) que se tiene al respecto, si se ha adquirido formación, si se utiliza y/o se tiene interés por formarse y aplicar en la práctica diaria, etc. Dentro de este mismo bloque, se incluye una sub-pregunta de carácter abierto para evidenciar que el conocimiento que se tiene del concepto corresponde con las aplicaciones que le dan en el aula, si fuera el caso. Del mismo modo, al finalizar el cuestionario se añade un apartado de sugerencias y/o observaciones.

Respecto a la validación, se ha utilizado la validación basada en el juicio de tres expertos (en validación y en cuestionarios) de la Facultad de Magisterio de la Universitat de València y, la validación de caso único, donde el cuestionario fue aplicado a expertos en el ámbito educativo, con las mismas características a los de la muestra y, además, uno de ellos formado y experimentado en gamificación. En ambos casos se realizaron revisiones críticas siguiendo los criterios de verificación de adecuación de las preguntas con relación a los objetivos marcados, equilibrio y armonía de la estructura general, detección de la ausencia de preguntas o de elementos graves y adecuación de la longitud. Asimismo, dichos expertos realizaron comentarios y propuestas de mejora sobre el cuestionario en los dos formatos propuestos.

Los participantes son 58 maestros especialistas de música de los centros educativos de la comarca horta oest de la Comunidad Valenciana que ofrecen educación primaria en el curso escolar 2018-2019. Debido al desconocimiento de la acogida que iba a tener por parte de dichos docentes, se planteó no limitar la zona de estudio inicialmente y esperar a valorar el número y la población de la que se obtenían más respuestas en el periodo de 15 días para acotar la zona, de esta manera ceñir el tamaño de la muestra al tiempo del que se dispone. El cuestionario se 
diseñó mediante Formularios-Google y se les hizo llegar en formato on-line a través de los correos corporativos. A lo largo de dos semanas se obtuvieron 34 cuestionarios, la gran parte de ellos eran de pueblos/ciudades de la comarca horta oest, motivo principal por el que se decidió limitar la muestra a esta zona. Tras el proceso de recogida, para el estudio se cuenta con un total de 58 cuestionarios, de los 83 obtenidos.

Para promover que los docentes respondieran con facilidad, se fue contactando con los centros por teléfono y por correo, de esta manera se informaba sobre el estudio, se confirmaban la cantidad de docentes especialistas que hay en el centro y se ofrecía la posibilidad de elegir la vía que les resultaba más cómoda para contestar al cuestionario. Si a los 15 días (aproximadamente) no se había obtenido respuesta se volvía a contactar con ellos para avisar de la entrega y/o recogida del sobre con el cuestionario. En definitiva, el cuestionario se les ha proporcionado, mayoritariamente, por correo y en sobre a la dirección del centro.

En segundo lugar, se ha creado un cuadrante con todos los centros educativos de educación primaria de la provincia de Valencia, señalando la comarca de la que formaba parte e incluyendo los datos de contacto, de esta forma se hace seguimiento de los avances. El siguiente paso fue analizar los datos obtenidos. Y, finalmente, se pasó a la interpretación de los resultados.

Los instrumentos que se han utilizado para llevar a cabo el estudio han sido numerosos y variados, no obstante, destacar algunos imprescindibles como el ordenador con conexión a internet, listado de los centros educativos de educación primaria de la Comunidad Valenciana por zonas (con direcciones y teléfonos), dispositivos digitales y aplicaciones de comunicación, vehículo propio para acceder a los centros, cuestionarios con impresión a color en folios A3, sobres de tamaño $A 3$, convertidor de códigos $Q R$ y herramientas de edición y procesamiento de textos (Pages y Word). La herramienta utilizada para los análisis de resumen y descripción estadística ha sido el software R-UCA.

\section{RESULTADOS}

Recordando lo especificado en el apartado anterior, se han utilizado los resultados de 58 cuestionarios, una muestra superior a 40, por lo que inicialmente se puede afirmar que las variables de estudio se comportan como una distribución "normal". Por otra parte, tras contemplar los presentes análisis y realizar las diferentes pruebas de contrastes entre las variables de clasificación, cabe destacar que las diferencias entre estas no son lo suficientemente significativas, por lo que se determina (con un 95\% de seguridad) que no existen evidencias estadísticas claras para confirmar que las variables presentadas sean determinantes en los resultados obtenidos. De modo que los datos que se muestran a continuación tienen carácter general. 
El primer objetivo que se planteó fue comparar el uso de los juegos como herramienta de desarrollo y evaluación de los contenidos curriculares de música frente a otras estrategias. En las figuras 1 y 2, se muestran la frecuencia con la que los docentes especialistas utilizan en sus clases las diferentes técnicas para trabajar y evaluar los contenidos curriculares.

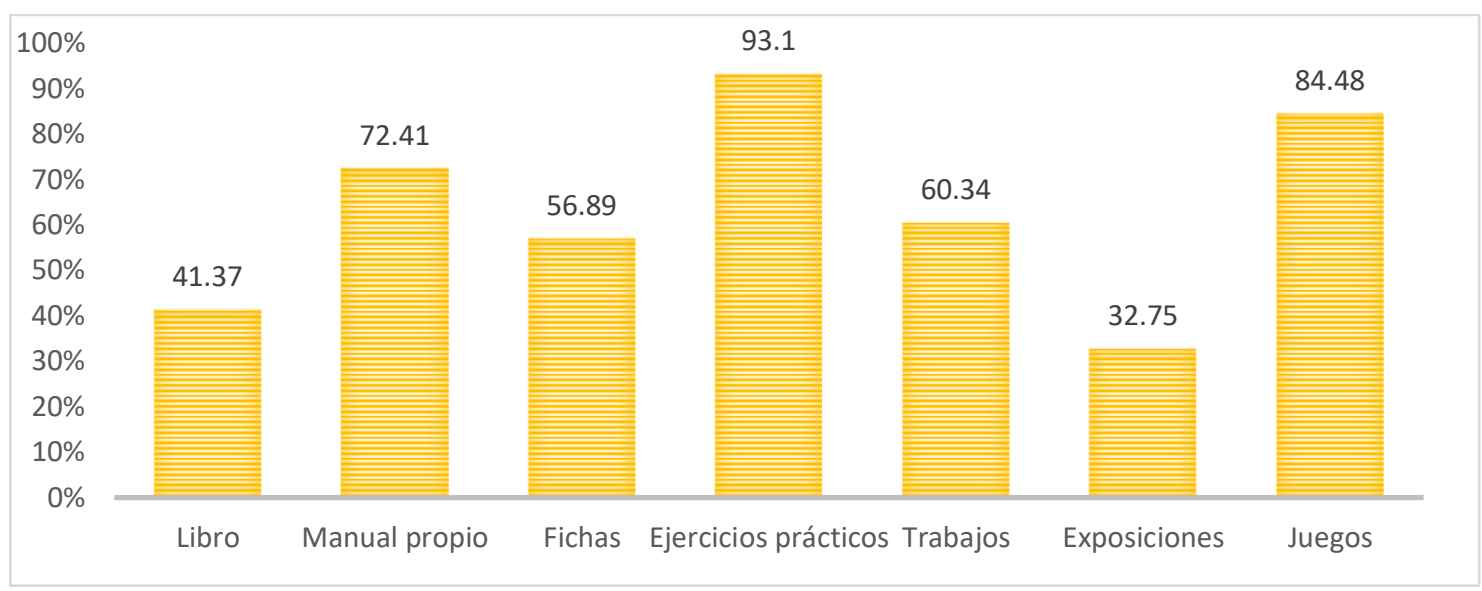

Figura 1. Frecuencia de uso de las diferentes técnicas para trabajar los contenidos

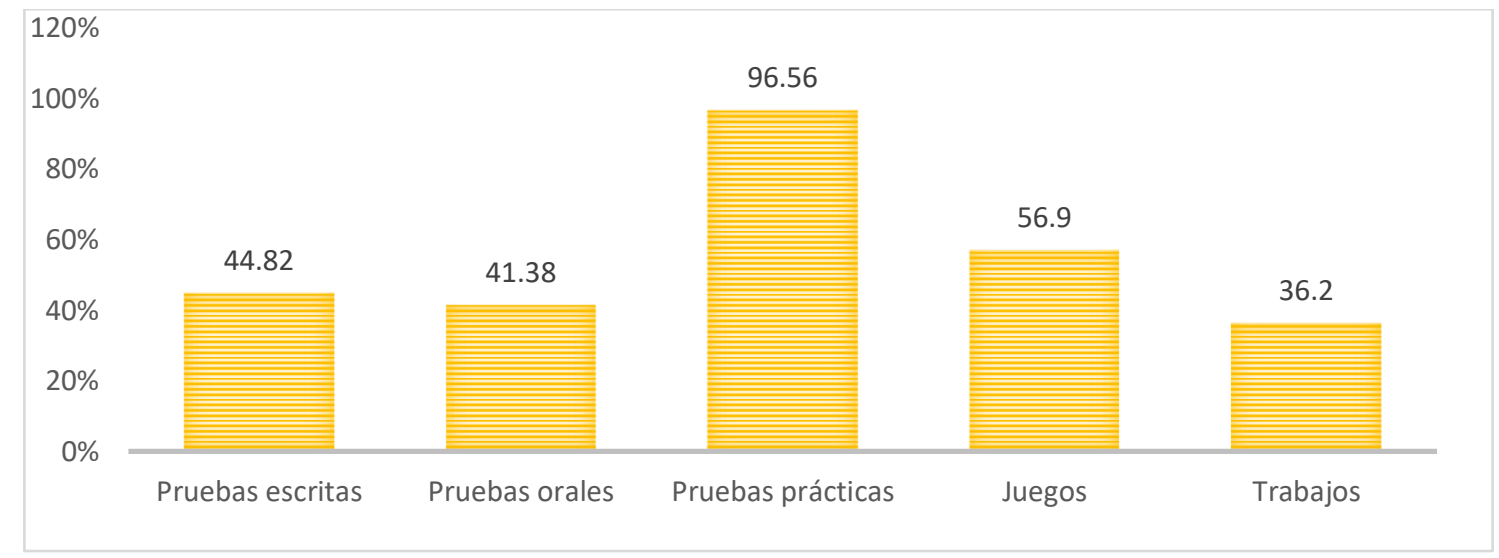

Figura 2. Frecuencia de uso de las diferentes técnicas para evaluar los conocimientos y los contenidos

En primer lugar, como se puede observar, los ejercicios prácticos y los juegos son las técnicas más utilizadas en las sesiones de música, siendo el 93,1\% y el 84,48\%, respectivamente, la cantidad de maestros/as que aplican dichas técnicas. Casi la totalidad de los maestros. El 72,41\% de los docentes diseñan un manual propio para guiar las sesiones, el 60,34\% realizan trabajos, el $56,89 \%$ fichas y el $32,75 \%$ proponen exposiciones para el desarrollo de contenidos. Destacando el $41,37 \%$ de participantes que siguen utilizando el libro como herramienta de trabajo en sus sesiones, es decir, poco menos de la mitad de los docentes. 
En segundo lugar, el 96,56\% de los participantes declaran realizar pruebas prácticas para evaluar, evidenciando así ser la técnica más empleada entre los docentes. Los juegos son utilizados por más de la mitad de los participantes (56,9\%), el uso de pruebas escritas y orales siguen estando presentes dentro de las aulas con el $44,8 \%$ y el 41,4\%, respectivamente. Por último, el 36,2\% de los docentes declaran utilizar trabajos como medio para evaluar los conocimientos de los discentes. Que las pruebas escritas estén presentes en casi la mitad de los casos, se ve influenciado por corresponder ante el sistema de evaluación que siguen los centros con el que los profesores deben calificar numéricamente a sus alumnos, como expone el $62 \%$ de los encuestados. A lo largo de los años, este se ha convertido en el método más satisfactorio para justificar los resultados a las familias e, incluso, alumnos (Cañadas, Santos-Pastor y Castejón, 2018).

A continuación, centrándonos en el segundo objetivo propuesto, se muestra de manera general el conocimiento del concepto (figura 3) y la formación (figura 4) que poseen los docentes en relación con la gamificación:

CONOCIMIENTO

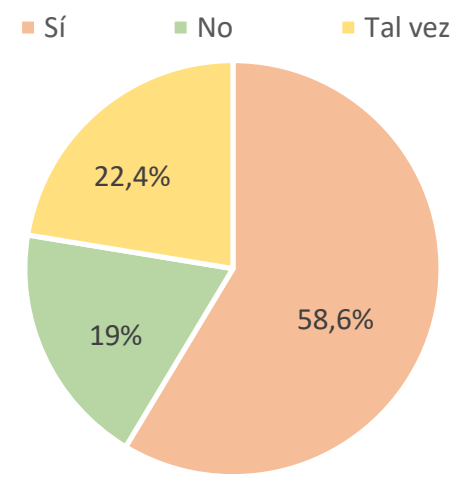

Figura 3. Conocimiento sobre gamificación
FORMACIÓN

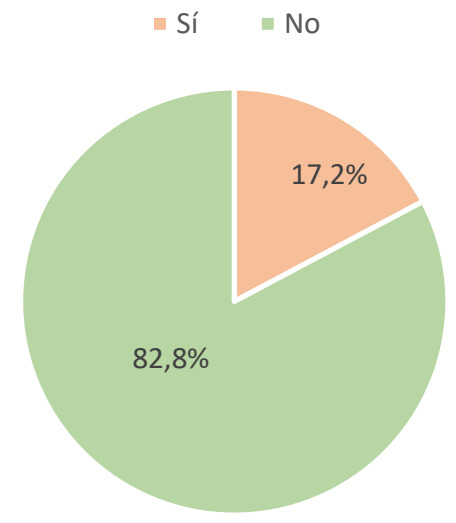

Figura 4. Formación sobre gamificación

Casi el $60 \%$ de los participantes afirman conocer la definición del concepto. Al 22,4\% les suena, pero no aseguran saberlo correctamente. Y, el 19\%, declaran no haber escuchado nunca sobre la presente estrategia. Tan solo el 17,2\% de los participantes confirman haber recibido formación al respecto. A continuación, y en el mismo cuestionario, se les facilita una breve explicación escrita de lo que consiste la gamificación para que todos puedan contestar, lo más objetivamente posible, si aplican o no dicha estrategia en sus sesiones. En la figura 5 se muestran las respuestas obtenidas y en la figura 6 se compara la formación previa de los maestros que aplican la estrategia: 


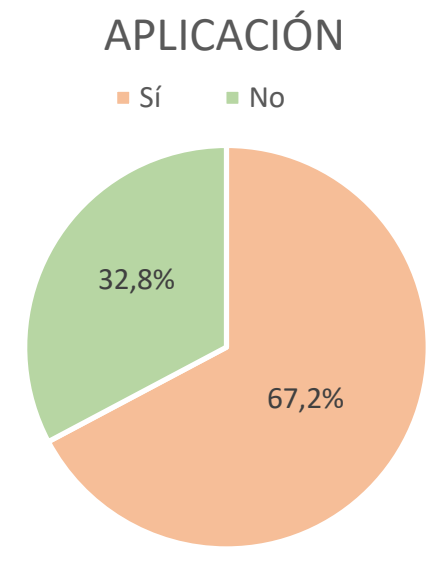

Figura 5. Aplicación de la gamificación en las aulas de los participantes

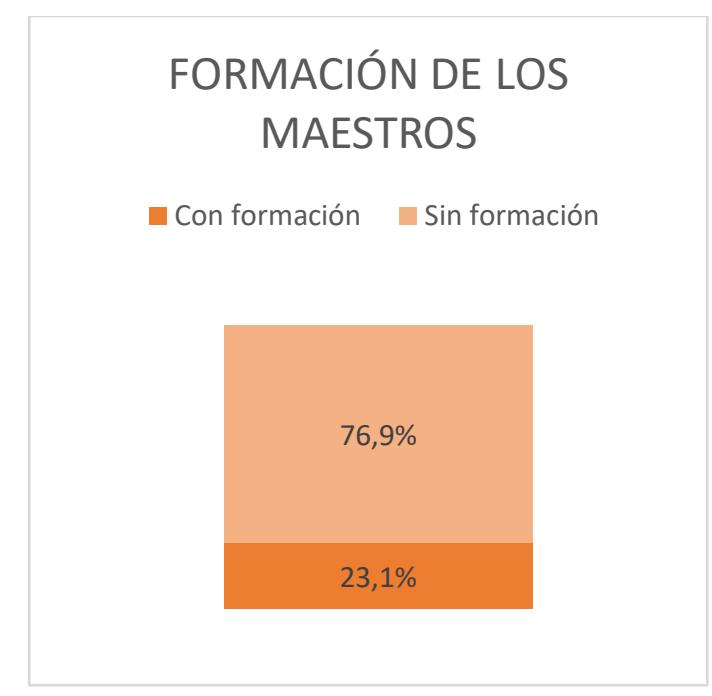

Figura 6. Formación de los maestros que aplican la gamificación en sus aulas

Independientemente de si han obtenido formación o no, el 67,2\% de los encuestados afirman aplicarla en su día a día, y tan solo el 23,1\% de estos tienen formación previa. Es decir, que el 76,9\% de los encuestados (más de la mitad) aplican la estrategia sin previa formación. Para conocer la fiabilidad de los resultados obtenidos en este apartado y valorar la importancia de la continua formación docente para la aplicación de herramientas con éxito, se les solicita algún ejemplo de prácticas y experiencias gamificadas que realizan en el aula. Siguiendo las anotaciones de los expertos en gamificación como Kapp (2012) y Cordero (2015), al analizar los 
ejemplos que ofrecieron, se puede observar en los participantes encuestados que utilizan la gamificación que solo el 38,4\%, adoptan prácticas que se adecuan a dicha estrategia (incluyendo el 23,1\% con formación), el 20,5\% ejemplifican ejercicios a los que serían necesarios añadir una explicación más detallada para poder calificar las experiencias como gamificación, y el 35,8\% de las respuestas no corresponden a lo que se entiende por gamificación. El 5,1\% de los encuestados dejaron en blanco esta cuestión.

Asimismo, los encuestados quienes aplican la gamificación dentro de sus aulas, aseveran que encuentran una serie de beneficios que se resumen en: motivación, diversión, aumento del interés por la materia y los contenidos, aprendizaje significativo, inclusión y competitividad entre los participantes, desarrollo de habilidades, retención de contenidos y dinamismo en las sesiones. En cambio, del 33\% de los participantes que afirman no incluir dicha estrategia en su práctica docente lo justifican con los motivos expuestos en la figura 7:

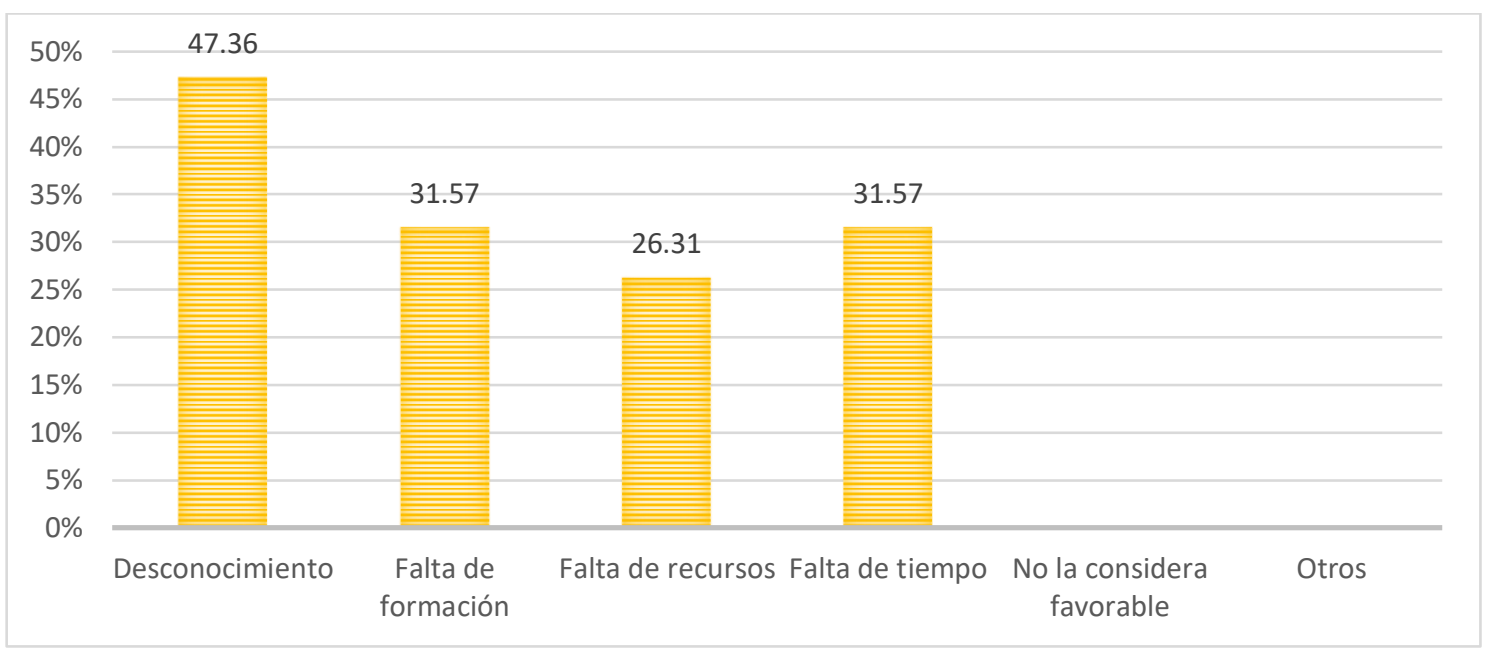

Figura 7. Motivos por los que los docentes no aplican la gamificación

El $47,4 \%$ confiesa que es por desconocimiento al concepto, el 31,6\% no la aplican por falta de formación y de tiempo, y el 26,3\% por falta de recursos.

Respecto al último objetivo planteado en el estudio, solo el 49\% afirma tener interés en emplear la gamificación. $Y$, entre las dificultades que encuentran los docentes para poner en práctica determinadas metodologías en sus aulas de música, como puede ser la gamificación o los juegos, se destaca la formación con el $84,2 \%$, seguido de la falta de tiempo $(52,6 \%)$ y los recursos $(36,8 \%)$. El espacio, las ideas, la colaboración del centro y de las familias se encuentran por debajo del $30 \%$. 


\section{DISCUSIÓN Y CONCLUSIONES}

Teniendo en cuenta la complejidad de generalizar que conlleva las investigaciones que se realizan a un grupo de personas concretas, en un momento determinado, la pretensión del presente estudio es dar a conocer el papel que cumplen los juegos y la gamificación en las aulas de música de los centros de Educación Primaria de l'horta Oest de Valencia en el presente curso (20192020), partiendo de los intereses y las prácticas de los docentes. En este punto, tras haber analizado los cuestionarios realizados a los docentes participantes se llega a 3 conclusiones principales.

El uso del juego destaca entre las técnicas consideradas como tradicionales, como son los libros y el uso de fichas. Por lo que, el principal componente que fundamenta la gamificación, es decir, el uso de técnicas y elementos de los juegos para motivar al alumnado (Kapp, 2012), están presentes en el $84,5 \%$ (en el caso de que el objetivo sea trabajar contenidos) y en el 57\% (si el objetivo es evaluar los aprendizajes) de las aulas de música, aunque el objetivo principal no sea gamificar. Es decir, que un gran porcentaje de docentes apuesta por la inclusión de los juegos en sus sesiones. Moyles expresa que el juego es un recurso que, bien aplicado, promueve el éxito en cualquier etapa y materia, a corto y a largo plazo (1999). De esta manera se puede explicar que los docentes, sobre todo aquellos con una categoría profesional con estabilidad incierta como sustitutos o interinos, apliquen métodos que aseguren una recepción positiva rápida por parte del alumnado y al mismo tiempo mantengan la calidad educativa. Esto fundamentaría también la destacable inclusión del juego en las programaciones de los docentes en la actualidad y la disminución, aunque lenta, del uso del libro para trabajar los contenidos. No ocurre lo mismo a la hora de evaluar, lo cual deja entrever que se desconoce el potencial del juego como técnica de evaluación de conocimientos y de contenidos.

No obstante, con relación a la gamificación, a la segunda conclusión a la que se llega es que la formación de los docentes al respecto todavía es insuficiente para poder llevarla a las aulas de manera exitosa. A pesar de que más de la mitad (59\%) de los encuestados afirman tener conocimientos sobre gamificación y el 67\% confirma aplicarla en sus aulas, el $83 \%$ de los encuestados no ha obtenido nunca formación al respecto, lo cual influye en las representaciones equivocas o vagas que demuestra un gran porcentaje $(64,1 \%)$ de los docentes que aplican la gamificación en sus aulas, según los ejemplos que exponen en los cuestionarios. Una gran parte de estos ejemplos dejan entrever que la confusión proviene de las similitudes que guarda esta estrategia con el ABJ o con la idea de realizar actividades lúdicas para fomentar el aprendizaje activo, como método de enseñanza que involucra activamente al alumnado en el aprendizaje, para que sean ellos propios protagonistas del proceso (Schwartz y Pollishuke, 1995). Todos tienen el juego como núcleo de la metodología, pero las finalidades son diferentes. Como afirma Riera, "no todo lo que sea añadir un juego a algo aburrido es gamificación" (por UOC, 2018). Gran parte de los docentes que dicen incluir la estrategia en sus programaciones, muestran tener interiorizados mitos o falsas informaciones en torno a la presente estrategia. La mayoría tienen la 
tendencia a confundir el juego serio con la ludificación. Aunque ambos tienen el juego como base, es importante recordar que el primero se trata de un recurso que tiene el propósito de enseñar y aprender, mientras que el segundo busca utilizar aquellos mecanismos de los juegos que motivan a los participantes para crear experiencias con la finalidad de generar acciones concretas (Marczewski, 2013). Debido a diversos estudios que señalan a la gamificación como la "llave de una mayor productividad y de un aprendizaje más profundo" (Meneses, 2019), hoy en día se pueden encontrar cursos de formación y artículos académicos que ofrecen a los docentes, y a cualquier otro lector, los conocimientos necesarios para profundizar en el tema. Sin embargo, y a pesar del interés que muestran algunos de los docentes, tan solo un $17 \%$ ha recibido formación al respecto, lo cual hace reflexionar y llegar a una conclusión más concreta dentro de este apartado: la formación o las vías de acceso a la información, no son los adecuados para facilitar y motivar al docente en formarse en el tema. Como explica Gaitán, ser docente es trascender en la nueva y generadora sociedad, investigando y formándose constantemente para dar una respuesta inmediata a las necesidades del alumno actual, sin caer en la tentación de mantener falsas creencias ligadas a la propia experiencia (2018). En esta línea cabe mencionar la seguridad que caracteriza a los docentes, sobre todo a la hora de aplicar diferentes estrategias sin tener adquiridos conocimientos suficientes para dar respuesta a los problemas que surgen a diario en el aula. En este sentido, se puede entender el alto porcentaje de docentes que utilizan en este caso la gamificación, a pesar de no haber recibido nunca ningún tipo de formación. De aquí la importancia de generar investigación en torno al presente tema y hacerlo llegar a los centros y a los docentes, para animarlos a formarse y a aplicar en sus aulas estrategias fundamentadas con resultados reales y para que, de esta manera, puedan crear sus propios juicios al respecto. Asimismo, recalcar la necesidad de adaptar los cursos formativos a los contextos de los maestros. Como señalan Fandos y Cano (2014), en el contexto de una sociedad compleja y con permanentes cambios, la formación docente debe ejercer un papel esencial, y por ello la exigencia de flexibilizar los proyectos formativos.

Pero, independientemente de los conocimientos y formación que tengan o no los especialistas, la incorporación de estrategias basadas en el juego y bien gamificadas ofrece beneficios en el proceso de enseñanza-aprendizaje. De aquellos maestros que sí aplican la estrategia en sus sesiones no hay nadie que exprese desagrado o resultados desfavorables, más bien al contrario, todos resaltan el aumento de la motivación, de la participación, de la atención y del interés de los alumnos hacia la materia tras realizar dichas experiencias, ya sea por el uso de herramientas que guían la actitud como ClassDojo como otras para evaluar conocimientos, por ejemplo, Kahoot. Estos son algunos ejemplos de los muchos que muestran los docentes encuestados, pero como afirma Arnedo en la entrevista realizada por UOC (2018), ya desde hace años se integran en la escuela herramientas que hacían más apetecibles realizar ciertas tareas y que se pueden relacionar con gamificación, como el uso de caras sonrientes para premiar actitudes o ejercicios superados, pegatinas que indican la comprensión de conceptos, etc. Pero gamificar no basta con aplicar el patrón PBL (Points, Badgets and eaderboards) y, por ello, la importancia de indagar y formarse de la que hablábamos anteriormente. 
Gracias a la participación tan favorable de los docentes, se conoce que además de la falta de formación, otra de las mayores dificultades que encuentran en su día a día para poder poner en práctica estrategias novedosas como es la gamificación, es la falta de tiempo. Tal y como señala Informe GEM, uno de los peligros de aumentar la carga de trabajo de los maestros es que estos no tengan tiempo para formarse y no se les permita enseñar en calidad a las demandas del alumnado y "en sistemas sumamente exigentes se puede intensificar la frustración" (2017, p.77). Los maestros son conscientes de la necesidad de formarse $y$, además, muestran interés por aplicar estrategias motivadoras, pese a que el tiempo y la formación dificulten llevarlo a la práctica, por ello la necesidad de acercar los estudios y resultados que se obtienen de investigaciones a sus contextos más próximos. Sería fundamental también, que el plan de estudio de los diferentes grados en maestros contemple la formación en metodologías e innovación docente, incluyendo diversas investigaciones que legitimen los resultados de incluir dichas metodologías y, de este modo, dar a conocer de manera actualizada la gran variedad de recursos que se pueden utilizar en el aula.

En definitiva, pese a que la gamificación se encuentra entre las herramientas educativas más sonadas actualmente, como se hablaba al inicio del estudio, este efecto no correspondería con la realidad educativa que se vive en las aulas de música de Educación Primaria. Sin embargo, no sucede lo mismo con el fenómeno de los juegos que, sin duda, continúa asumiendo un gran peso en las programaciones de los maestros. Asimismo, se pueden afirmar que se cumplen las hipótesis planteadas en el presente estudio.

Para concluir este apartado, cabe mencionar las dos limitaciones principales que se han encontrado a lo largo del desarrollo del presente estudio. Por una parte, la contradicción de algunas de las respuestas de los participantes en los cuestionarios. Por ejemplo, algunos confirmaban no aplicar la gamificación al mismo tiempo que confesaban que utilizarla traía beneficios en el aula. Asimismo, tras analizar las muestras de las prácticas gamificadas que realizan los docentes dentro de sus aulas, se comprueba que no coinciden con el concepto de gamificación. Esto sucede debido a que las respuestas generalmente conllevan una serie de sesgos que afectan a la veracidad de los resultados. Una posibilidad es que en las respuestas entran en juego sus creencias basadas en la experiencia sin tener una formación previa al respecto. Otras posibilidades es que las respuestas de los participantes pueden verse influidas por el sentimiento de aceptación profesional o que respondieran según lo que creen que el investigador quiere leer o según lo que les gustaría que fuera la realidad, aún sabiendo que el cuestionario era anónimo. De la misma forma, también existe la posibilidad de que no hayan comprendido correctamente las preguntas y eso también justificaría algunas de las respuestas confusas o poco argumentadas. $Y$, por otra parte, el tamaño muestral de los cuestionarios realizados a los docentes también ha sido una limitación. A pesar de haber conseguido una muestra de 58 maestros, estos no han sido lo suficientemente representativos del conjunto de la población para poder inferenciar con un 95\% de seguridad en algunos casos, como la relación 
entre variables, y mostrar así la situación actual de la gamificación en las aulas de música de educación primaria.

\section{REFERENCIAS}

Cordero, C. (2015). Gamification [Mensaje en un blog]. Agorabierta. Recuperado de https://www.agorabierta.com/2015/12/gamification/

De Soto, I.S. (2018). Herramientas de gamificación para el aprendizaje de Ciencias de la Tierra. Edutec. Revista Electrónica de Tecnología Educativa, (65), 29-39. https://doi.org/10.21556/edutec.2018.65.1143

Díaz, J., y Troyano, Y. (2013). El potencial de la gamificación aplicado al ámbito educativo. En III Jornadas de Innovación Docente. Innovación Educativa: respuesta en tiempos de incertidumbre. Sevilla, España: Facultad de Ciencias de la Educación de Sevilla.

Fandós, M., y Cano, J. (2014). Formación a distancia y retos actuales en los roles docentes y su vinculación con la empresa: propuesta y controversias. Edutec. Revista Electrónica De Tecnología Educativa, (45), 1-13. https://doi.org/10.21556/edutec.2013.45.14

Gaitán, Á. (2, Junio, 2018). Importancia de la formación docente en la actualidad [Mensaje en un blog]. Nexos. Recuperado de https://educacion.nexos.com.mx/?p=12855

Gallardo-López, J., y Gallardo-Vázquez, P. (2018). Teorías sobre el juego y su importancia como recurso educativo para el desarrollo integral infantil. Hekademos: revista educativa digital, (24), 41-51. Recuperado de https://www.hekademos.com/index.php/hekademos/issue/view/24-junio-2018

Gallardo, P., y Fernández, J. (2010). El juego como recurso didáctico en Educación Física. Argentina: Editorial Wanceulen.

Gómez-Martín, M., Gómez-Martín, P., y González-Calero, P. (2004). Aprendizaje basado en juegos. ICONO 14, Revista de comunicación y tecnologías emergentes, 2(2), 1-14. https://doi.org/10.7195/ri14.v2i2.436

Hamari, J., Koivisto, J., y Sarsa, H. (2014). Does gamification work? A literature review of empirical studies on gamification. En Hawaii international conference on system sciences (HICSS) (pp. 3025 - 3034). IEEE.

Informe de Seguimiento de la Educación en el Mundo 2017/2018. (2017). Rendir cuentas en el ámbito de la educación: cumplir nuestros compromisos. París, Francia: UNESCO. Recuperado 
http://unesdoc.unesco.org/in/rest/annotationSVC/DownloadWatermarkedAttachment/at tach import 89045273-020f-4007-af63$1 \mathrm{f} 4062 \mathrm{~b} 88 \mathrm{bb5} ?=261016 \mathrm{spa} \cdot \mathrm{pdf \& to}=545 \&$ from $=1$

Jenkins, H. (21, Marzo, 2011). Their Game: An Interview with James Paul Gee (Part One) [Mensaje en un blog] Consultado el 27 de enero de 2019 en http://henryjenkins.org/2011/03/how learners can be on top of.html

Kapp, K. (2012). The Gamification of Learning and Instruction: Game-based Methods and Strategies for Training and Education. San Francisco: John Wiley \& Sons.

Manzano, A., y Domínguez, J. (2018). Gamificación en la educación secundaria. En LópezMeneses, E., Cobos-Sanchiz, D., Martín-Padilla, A. H., Molina-García, L. y Jaén-Martínez, A. Experiencias pedagógicas e innovación educativa. Aportaciones desde la praxis docente e investigadora. Barcelona, España: OCTAEDRO, S.L.

Marczewski, A. (2013). Gamification: a Simple Introduction. Reino Unido: Editorial Lulu.

Cañadas, L., Santos-Pastor, Mạ. L., y Castejón, F.J. (2018). Evaluación en la formación inicial: ¿avance o retroceso? Bordón. Revista de pedagogía, 70(4), 9-22. https://doi.org/10.13042/Bordon.2018.64434

Meneses, M., y Monge, M. (2001). El juego en los niños: enfoque teórico. Revista Educación, 25 (2), 113-124. Recuperado de https://www.redalyc.org/pdf/440/44025210.pdf

Meneses, N. (7, agosto, 2019). La gamificación, llave de una mayor productividad y de un aprendizaje más profundo. El País. Consultado el 7 de agosto de 2019 en https://elpais.com/economia/2019/08/07/actualidad/1565167852 253423.html

Montero, B. (2017). Aplicación de juegos didácticos como metodología de enseñanza: Una Revisión de la Literatura. Pensamiento Matemático, 7(1), 75-92. Recuperado de https://dialnet.unirioja.es/servlet/articulo?codigo=6000065

Morillas, C. (2016). Gamificación de las aulas mediante las TIC: un cambio de paradigma en la enseñanza presencial frente a la docencia tradicional (Tesis doctoral). Universidad Miguel Hernández, Elche. Recuperado de: https://dialnet.unirioja.es/servlet/tesis?codigo=62213

Moyles, J. (1999). El juego en la educación infantil y primaria (Vol. 16). Madrid, España: Ediciones Morata.

Schwartz, S., y Pollishuke, M. (1995). Aprendizaje activo: una organización de la clase centrada en el alumnado (Vol. 134). Madrid: Narcea Ediciones. 
Torres-Toukoumidis, Á., y Romero-Rodríguez, L. (2018). Gamificación en Iberoamérica. Experiencias desde la Comunicación y Educación. Cuenca, Ecuador: Editorial Universitaria Abya-Yala

UOC. (29, Enero, 2018). Las claves de la gamificación, según Joan Arnedo y Daniel Riera [Mensaje en un blog] Consultado el 30 de diciembre de 2018 en https://x.uoc.edu/es/actualidad/las-claves-gamificacion-segun-joan-arnedo-y-danielrieral

Zichermann, G., y Cunnigham, C. (2011). Gamification by Design: Implementing Game Mechanics in Web and Mobile Apps. Cambridge, MA: O'Reilly Media.

\section{Para citar este artículo:}

Botella Nicolás, A. M., y Cabañero Castillo, E. (2020). Juegos y gamificación en las aulas de música de educación primaria. Edutec. Revista Electrónica De Tecnología Educativa, (73), 174188. https://doi.org/10.21556/edutec.2020.73.1755 